\title{
El lugar de las enmiendas del Pinciano a la Historia Natural: aciertos únicos, propuestas olvidadas, nuevas soluciones
}

\author{
Francisco Javier Bran García \\ Universidad Complutense de Madrid \\ fbran@ucm.es \\ ORCID iD: https://orcid.org/0000-0003-2609-0692
}

\section{The Place of El Pinciano's Emendations on the Naturalis Historia: Unique Suggestions, Forgotten Approaches, New Solutions}

Las enmiendas del Pinciano (Hernán Núñez de Guzmán) a la obra de Plinio el Viejo supusieron en su época una revolución filológica a la que se fueron acogiendo diversos editores. Sin embargo, su empleo para las ediciones dista de ser uniforme. Algunas de sus propuestas, hoy aceptadas, cuentan con atribuciones erróneas y permanecen silenciadas, mientras que otras, que pueden proporcionar soluciones plausibles para pasajes controvertidos, cayeron en el olvido a raíz de un uso indirecto e incompleto de las mismas. En el presente artículo ubicamos las Obseruationes in loca obscura aut deprauata historiae naturalis (Salamanca, 1544-1545) en un contexto más amplio de comentaristas y recopilamos una selección de los mayores aciertos que contienen, junto con una relación de nuevas propuestas para replantear el contenido de diversos pasajes.

Palabras clave: Pinciano; Historia Natural; crítica textual; enmiendas.
The emendations of Hernán Núñez de Guzmán (el Pinciano) to Pliny the Elder's work meant a philological revolution in his time, and one embraced by several publishers for centuries. However, the usage made for editions was far from consistent. Some of his corrections, accepted today, are however wrongly attributed, thus remaining silenced. In turn, other emendations could provide modern editors with plausible solutions for controversial passages, but they have been forgotten as a result of an indirect, partial use of El Pinciano's annotations. In this article, El Pinciano's Obseruationes in loca obscura aut deprauata historiae naturalis (Salamanca, 1544-1545) are placed in a wider context of commentators, and a selection of his wisest choices is displayed, along with a compilation of new variants in order to propose a reconsideration for certain passages.

Key words: Pinciano; Naturalis Historia; Textual Criticism; emendations.

Cómo citar este artículo / Citation: Bran García, Francisco Javier 2020: «El lugar de las enmiendas del Pinciano a la Historia Natural: aciertos únicos, propuestas olvidadas, nuevas soluciones», Emerita 88 (2), pp. 277-302.

Copyright: (C) 2020 CSIC. Este es un artículo de acceso abierto distribuido bajo los términos de la licencia de uso y distribución Creative Commons Reconocimiento 4.0 Internacional (CC BY 4.0). 


\section{Los PRimeros comentaristas de Plinio El Viejo EN EsPaÑa}

La Historia Natural es un texto que ha tenido una pervivencia destacada en razón tanto de la cantidad de testimonios como de su diversa índole. A los trescientos siete ejemplares que han llegado a nosotros con contenido de dicha obra ${ }^{1}$ hay que sumarles los distintos tipos de manipulaciones del texto pliniano, en forma de compendios o resúmenes. Por último, contamos con los comentarios y su variedad de contenidos, desde aquellos que se centran en explicaciones ampliadas hasta los puramente filológicos ${ }^{2}$. Por supuesto, había asimismo soluciones intermedias.

Un comentarista de Plinio que gozó de temprana aceptación fue Hermolao Bárbaro $^{3}$. La excepcional difusión de sus propuestas en ediciones impresas concitó alabanzas de muchos, pero también críticas por parte de Hernán Núñez de Guzmán (el Pinciano, ca. 1478-1553) ${ }^{4}$, consciente como era de la transmisión imparable de errores en la lectio recepta. La historia de los comentaristas españoles de la Historia Natural no da comienzo, empero, con el vallisoletano, sino que arranca con Francisco López de Villalobos ${ }^{5}$ y su Glossa litteralis ${ }^{6}$. Antes que él, también en suelo hispano, Lucio Flaminio Sículo había publicado un Commentariolus in Plinium prohemium ${ }^{7}$. Entre Villalobos

${ }^{1}$ Sillig 1851-1856 menciona a lo largo de su obra ciento treinta manuscritos, con ciertos problemas de análisis. Detlefsen 1869, p. 285, eleva la cifra a unos doscientos materiales, tras lo cual se han venido contabilizando «más de doscientos» manuscritos con texto pliniano (Ernout 1950, p. 20). Hoy en día disponemos del catálogo de Borst 1994, pp. 360-374, que recoge trescientos siete manuscritos, incluyendo excerpta y calendarios con contenido de la Historia Natural.

${ }^{2}$ En ocasiones adoptaban la forma de cartas encabezando las ediciones. Un elemento revelador del contenido son los diversos nombres con que se presentan: glossa, explanatio, castigationes, emendationes, annotationes, obseruationes, commentarius y otros (Moure 2008, p. 210).

3 Su obra de mayor calado son las Castigationes Plinianae (Roma, 1492-1493).

${ }^{4}$ Son abundantes las obras que, a lo largo de los últimos cincuenta años, han contribuido a un conocimiento más profundo de este autor. De particular relevancia son las aportaciones de Asís 1974, Guzmán Arias 1991, Signes, Codoñer y Domingo 2001 y Codoñer 2011.

${ }^{5}$ Encontramos abundantes datos biográficos en su epistolario. Una bibliografía básica no debería prescindir de Fabié 1886, Friedenwald 1939, Calamita 1952, Granjel 1979, Tremallo 1991 y Arrizabalaga 2002.

${ }^{6}$ Glossa litteralis in primum et secundum Naturalis historie libros (Alcalá de Henares 1524). En realidad constituía una paráfrasis explicativa al texto original.

${ }^{7}$ Dicha obra fue publicada en Salamanca en 1504. Más información acerca del autor y su comentario puede encontrarse en Ramos 2001 y 2006. 
y el Pinciano afloraron en España dos comentaristas más: Juan Andrés Estrany, cuya obra quedó inédita, en versión manuscrita, y Pedro Juan Olivar Sin ser comentarios en sentido estricto, las obras de otros tres autores españoles del s. XVI contienen apreciaciones referentes al contenido de la enciclopedia de Plinio: se trata de Jerónimo Muñoz ${ }^{9}$, Francisco Hernández ${ }^{10}$ y Gerónimo de Huerta ${ }^{11}$.

Núñez de Guzmán, que conoció a Villalobos y llegó a cartearse con él con cierta acrimonia, es autor del primer comentario filológico al texto pliniano. En sus Obseruationes in loca obscura aut depranata historiae naturalis (Salamanca, 1544 y 1545) cubre toda la extensión de la Historia Natural salvo su primer libro, que contiene únicamente los índices y la carta dedicatoria, y el último. La razón que nos da para dejar fuera el libro XXXVII es muy elocuente: de los dos manuscritos de los que dispone, uno de ellos (el Salmanticensis, al que califica como semiuetus, hoy perdido ${ }^{12}$ ) no abarca más que hasta el libro XV, y el otro (el Toletanus, designado como uetustissimus ${ }^{13}$ ) se detiene en el libro XXXVI. Aunque accedió también a un texto zaragozano que le pidió a Zurita para poder concluir su tarea, lo recibió un tanto tarde y, en consecuencia, nunca llegó a publicar esa última parte. Hemos comprobado que, por lo menos, utiliza dicho manuscrito en tres ocasiones. Una nota de Sillig 1831-1836, p. v, xi ${ }^{14}$, llevó a Reeve $(2006$, p. 182) a concluir que lo empleó una sola vez, pues en vano buscó nuevas menciones a partir del libro XXVII. Sin embargo, el Pinciano no lo cita en el cuerpo de sus Obseruatio-

${ }^{8}$ Sus Annotationes se incluyen en la ed. parisiense de 1536.

${ }^{9}$ El astrónomo comentó antes de 1579 diversos pasajes de Nat. II (Baranda 2004, pp. 62-63).

${ }^{10}$ Autor de la primera traducción castellana. Aunque se suele dar tal distinción a Gerónimo de Huerta, ya que la obra de Hernández no llega a la imprenta hasta 1628, Somolinos y Nogués 1998 dan noticia de que la concluye en 1576.

11 Tras apariciones parciales, su traducción completa se publica en Madrid 1624-1629.

12 Nuestra búsqueda intensiva en registros modernos, inventarios antiguos y catálogos de los colegios mayores ha sido infructuosa.

${ }^{13}$ Hemos de ser cautelosos con tales designaciones, sobre todo teniendo en cuenta el desarrollo de la paleografía. Torre de Rezzonico 1763 hizo radicar el Toletanus en el s. IX, por lo que el manuscrito gozó del favor de Sillig 1831-1836 y de Ian 1870, quienes mostraban preferencia por los materiales uetustiores. Detlefsen 1866-1873 retrasa la fecha al s. XIII, y a partir de entonces pierde preponderancia en las ediciones. De acuerdo con lo dicho, el Salmanticensis debería haberse compuesto en torno al s. XIV.

${ }^{14}$ Caesaraugustanus, primum nominatus a Pintiano ad 27, 4. §. 20. 
nes, sino en una parte final dedicada a los errores librariorum magis insignes correcti, et loca quaedam retractata, y a partir de ahí recurre a él tres veces. Tras un examen intensivo podemos reforzar su identificación con el actual escurialense V.I.14, uno de cuyos exlibris nos informa de que, antes de llegar a manos de Zurita, había estado en la cartuja de Aula Dei, un monasterio sito en Zaragoza ${ }^{15}$. El Escorial alberga otros dos textos plinianos: uno, Q.I.4, carece de vínculo conocido con Zurita; otro, R.I.5, de gran parecido con V.I.14, muestra varias manecillas al margen, que no parecen obra del Pinciano ${ }^{16}$.

Volviendo a Núñez de Guzmán, la validez de sus Obseruationes se ha visto reforzada por la presencia de algunas de sus lecturas en las ediciones posteriores, incluso en casos en que se trataba de una labor ope ingenii. Conocida tal relevancia, haremos un breve esbozo de las características de las enmiendas, para centrarnos seguidamente en una selección de las mismas ${ }^{17}$.

\section{Tipología de las enmiendas del Pinciano}

Formalmente, el esquema de las Obseruationes es muy simple: su autor indica un segmento de texto (según la distribución antigua de capítulos y parágrafos, presente en la ed. de Froben), escribe a continuación el fragmento que le interesa, de longitud variable, y añade sus reflexiones sobre el mismo. Entre frecuentes críticas a Hermolao y numerosas referencias a otras ediciones, autores y comentaristas, termina por señalar la variante que prefiere. En algunos casos, no obstante, mantiene cierta duda que espera que pueda juzgar el lector avezado.

El Pinciano combina para su trabajo diversas técnicas que se adelantan a la crítica textual. Por un lado, tuvo en cuenta las lecturas presentes en los manuscritos toledano y salmantino. Recurre asimismo a lo que él llama uetus lectio (una denominación bastante vaga que parece referirse a un consenso relativo de sus manuscritos) y a ediciones impresas previas a Hermolao. Concretamente

${ }^{15}$ F. 252re.: «Es del monesterio (sic) de Nuestra Señora de Aula Dei de la Cartuxa». Hasta hoy este libro no estaba localizado definitivamente: solo se apunta la posibilidad en Signes, Codoñer y Domingo 2001. Reeve 2006, p. 181, aporta algunos datos más para su identificación.

${ }^{16}$ Al ser material prestado y no de propiedad, dudamos de que él mismo las hubiese señalado. Por lo demás, hay marcas en libros que, hasta donde sabemos, no empleó para su estudio de la Historia Natural.

${ }_{17}$ Nos centraremos en algunos aspectos menos tratados en los estudios que, a partir del trabajo de Asís 1974, se han dedicado al Pinciano. 
tiene en sus manos algunas ediciones romanas, una de $\operatorname{Parma}^{18}$ y las lecturas de Marciano Capela ${ }^{19}$. Como cabe esperar, dota de preferencia a las variantes que contienen los manuscritos, pues considera que albergan lecturas, por lo general, más acertadas que las ediciones, pero tampoco aplica su criterio ciegamente.

Con una biblioteca bien pertrechada de obras clásicas, las auctoritates suponen una segunda herramienta valiosa para el humanista, algo especialmente útil en el caso de Plinio el Viejo, quien había hecho un compendio de informaciones de un gran volumen de fuentes. El Pinciano recurre a un número ingente de citas y las plasma con suficiente exactitud como para deducir que trabajó con los textos mismos y no únicamente de memoria. En este caso, y si bien deposita su confianza en los clásicos, se percata de que no era una técnica infalible (Nat. XV 84). Para ocuparse de los primeros quince libros de la Historia Natural, consulta nada menos que cien autores y editores, desde aquellos de la Antigüedad hasta otros más próximos a él e incluso estudiosos con quienes tuvo relación y que le hicieron llegar sus sugerencias para mejorar las enmiendas ${ }^{20}$. En medio de un extenso listado descuellan por su frecuencia de uso Aristóteles (en 168 ocasiones), Estrabón (69), Esteban de Bizancio (66), Ptolomeo (63), Teofrasto (55), Marciano Capela (37), Columela (29) y Varrón (19). Salta a la vista, por tanto, que es un perfil dominado por las fuentes de Plinio y, específicamente, aquellas que se traen a colación para los volúmenes de geografía, botánica y zoología. Como consideración añadida, indicaremos que con frecuencia consulta los textos griegos a través de traducciones, como las de Teodoro Gaza o Pedro Gil ${ }^{21}$. Esto no ha de tenerse por demérito, ya que la habilidad del Pinciano para localizar las fuentes griegas originales, así como el diálogo que entabla con los hombres

18 Tras investigar a fondo varias ediciones de Parma, comprobamos que podría tratarse de una de las impresiones de Esteban Corallo. La más antigua a la que se ha tenido acceso data de 1476. Al ser una obra que se reeditó en Parma varias veces, no hay más datos para saber si se trataba en concreto de esa edición o de otra posterior. Sin embargo, las lecturas del Pinciano coinciden con las cotejadas.

${ }^{19}$ Códices de la Historia Natural utilizados por Marciano Capela, de acuerdo con las lecturas que este autor presenta en su De nuptiis Philologiae et Mercurii y que recoge el Pinciano.

${ }^{20}$ Apolodoro, Higino, Ovidio o Tucídides coexisten junto a Esteban de Bizancio, Hesiquio de Alejandría o Isidoro de Sevilla, pero también con Cristóbal Oroscio, Honorato Juan, Láscaris Rhyndaceno y Masario.

${ }^{21}$ Pierre Gilles, latinizado Petrus Gillius o Gyllius, se ocupó junto con Conrado Gesner de la traducción de los De animalium natura libri XVII. 
de letras desde la Antigüedad hasta sus coetáneos son de todo punto dignos de encomio. Solo recurre al texto griego original en aquellos casos en los que no parecen convencerlo las versiones a su alcance (Nat. XII 35). De manera puntual se vale de alusiones poco específicas (Graeci auctores, geographi omnes) que complican nuestra labor de búsqueda de fuentes.

Finalmente, encontramos un buen número de enmiendas que propone desde su olfato filológico, como estudioso que de sólito lidiaba con textos latinos y que conocía a la perfección el estilo pliniano: hemos de recordar que fue profesor de la asignatura de Plinio el Viejo en la Universidad de Salaman$\mathrm{ca}^{22}$. En estas conjeturas que hace repugnantibus exemplaribus o contra omnes se guía, como decimos, por el usus scribendi, elementos de sintaxis y léxico, e incluso el contenido mismo. Si bien resultan discutibles, puesto que carecen de testimonios que las refrenden, hay casos en los que son las más acertadas y su uso se ha convertido en el canónico.

\section{SeleCCión DE ENMIENDAS}

\section{Método y disposición}

Para el presente trabajo hemos analizado todas las enmiendas del Pinciano hasta Nat. XV, pues es la extensión en la que dispone de dos manuscritos para llevar a cabo una labor filológica más completa. En total, se han estudiado más de dos mil, de las que empezaremos consignando las mal atribuidas. Nos detendremos, a continuación, en aquellas que son representativas de la sagacidad filológica del humanista por haber constituido variantes comúnmente aceptadas para el texto pliniano sin apenas apoyo documental, para terminar con una selección de enmiendas que pueden aportar lecturas válidas para el texto de Plinio, aunque hoy no se recogen en los aparatos críticos ${ }^{23}$.

${ }^{22}$ Leyó su cátedra de Griego desde 1523, y la de Plinio en 1526-1527 (Rodríguez-San Pedro 2012, p. 70). La existencia de una edición en formato de quince centímetros $\left(8 .^{\circ}\right)$ podría evidenciar un uso estudiantil. De dicha edición (Amberes, 1547) se conocían dos copias, en el British Museum y en la Bibliothèque Nationale (Nauert 1980, p. 394). A ellas sumamos dos más que hemos hallado en la Biblioteca Nacional de España (U/1560) y en la Zentralbibliothek de Zúrich (Alte Drucke, Magazin 06, signatura 20.375).

${ }^{23}$ Consignamos en primer lugar la lectura del pasaje de Mayhoff, a fin de proporcionar un contexto amplio que permita comprender el contenido; entre corchetes señalamos el volumen, 
Empleamos las siguientes siglas para los manuscritos ${ }^{24}$ :

A Leidensis Vossianus F4, s. IX (Leiden, Bibliotheek der Rijksuniversiteit)

C Cenomanensis 263, s. XII (Le Mans, Bibliothèque Municipale 263)

D Vaticanus Latinus 3861, ca. 800 (Vaticano, Biblioteca Apostolica Vaticana)

E Parisinus Latinus 6795, ss. IX-X (París, Bibliothèque Nationale)

$E^{S}$ Mano probablemente del s. XII. Parte de un ejemplar más antiguo que guardaría relación con $A$ y los excerpta Crikladensia ${ }^{25}$

F Leidensis Lipsii n. 7, s. IX (según Ernout 1947) o s. X (Serbat 1995) $(=f \text {, Leiden, Bibliotheek der Rijksuniversiteit })^{26}$

$R \quad$ Florentinus Riccardianus 488, ss. IX-X (Florencia, Biblioteca Riccardiana). Una segunda mano presenta lecturas de entre los ss. IX y XI

libro y capítulo de las Obseruationes, así como la página en que lo trata el Pinciano. Siguen las variantes textuales según la disposición propia de un aparato crítico positivo, ordenando las lecturas de más antiguas a más recientes. Se han unificado datos de las ediciones de Dalecampio, Harduino, Sillig, Detlefsen, Mayhoff, Rackham, Ernout, Einaudi y König-Winkler. Añadimos las lecturas del Pinciano y de la edición de Froben.

${ }^{24}$ Aportamos una relación sumaria, con la fecha de composición y la ubicación actual, para evitar una extensión excesiva. En general, respetamos las designaciones presentes en Teubner y Les Belles Lettres. En caso de disensión, se señalará convenientemente. En cuanto a las ediciones, van notadas como sigue: Bar. (Castigationes de H. Bárbaro, Roma, 1492-1493), Frob. (Froben, Basilea, 1539), Gel. (Gelenio, Basilea, 1544), L. (ed. de Lion, I. N. Victorio 1563), Hard. (Harduino, París, 1685), Brot. (Broterio, París, 1779), Sil. (Sillig, Hamburgo y Gotha, 1851), Det. (Detlefsen, Berlín, 1866 y 1904, con especificación en superíndice cuando no coinciden ambas ediciones). MCap se refiere a las lecturas que el Pinciano encuentra en De nuptiis Philologiae et Mercurii de Marciano Capela. Las ediciones modernas se marcan como May. (Mayhoff, Stuttgard, 1892-1909), Rack. (Rackham, Londres y Cambridge, 1938), Ern. (Ernout, introductor del primer vol., París, 1950, designa la colección de Les Belles Lettres), Ein. (Einaudi, Turín, 1982-1984) y KW (König, R. y Winkler, G, Dusserldorf y Zúrich, 1974-1996). Con edd. nos referimos a todas las ediciones modernas a partir de la de Detlefsen, mientras que uet. son las ediciones antiguas, edición vulgata. Un signo de exclamación señala una lectura errónea en el editor.

${ }^{25}$ Compuestos por Roberto de Cricklade para Enrique II de Inglaterra a finales del s. XII. La obra, titulada Defloratio naturalis historiae Plinii Secundi, abarca toda la Historia Natural con la usitada excepción del libro I. Cuenta con edición reciente de Näf 2003.

${ }^{26}$ La identificación de $F$ con el anteriormente conocido como Chiffletianus $(f)$ fue defendida por Detlefsen 1904, p. viii, y recogida por Ernout 1947, p. 31. Walter 1996 lo prueba suficientemente, y Reeve 2007, p. 124, lo confirma. 
$S \quad$ Salmanticensis, testimonio conjetural ${ }^{27}$

$T \quad$ Toletanus, s. XIII (Madrid, Biblioteca Nacional, 10042)

$V \quad$ Vossianus Latinus folio 61, ca. 800 (Leiden, Bibliotheek der Rijksuniversiteit)

X Luxemburgensis 138, ca. 1150 (Luxemburgo, Bibliothèque Nationale)

a Vindobonensis CCXXXIV, ss. XII-XIII (Viena, Österreichische Nationalbibliothek, Latinus 9-10)

d Parisinus Latinus 6797, finales del s. XII o inicio del s. XIII (París, Bibliothèque Nationale)

e Parisinus Latinus 6796.A, copia de E del s. XIII (Serbat 1995) (París, Bibliothèque Nationale)

$g \quad$ Parisinus Latinus 6800 (Colbertinus I en Sillig), finales del s. XII (París, Bibliothèque Nationale)

$h \quad$ Parisinus 6801, s. XV (París, Bibliothèque Nationale)

$l \quad$ Arundelianus 98, quizás del s. XII (Londres, British Library)

p Pollinganus Latinus 11301, antes de 1459 (Múnich, Bayerische Staatsbibliothek)

$x \quad$ Añadidos a $X$ que completaban el manuscrito, aparentemente anteriores

Ch Neo-Eboracensis M.871 $1^{28}$, primera mitad del s. IX ${ }^{29}$ (Nueva York, Pierpont Morgan Library)

Co Kobenhavnensis Reg. $212 f^{o}$, s. XII (Copenhague, Kongelike Bibliotek, Gamle Kongelige Samling)

Colb $^{30}$ Parisinus Latinus 6796 A, ss. IX-X (Colbertinus II en Sillig) (París, Bibliotèque Nationale)

Ox Oxoniensis 274, s. XII (Oxford, New College)

${ }^{27}$ Ernout emplea $s$ para designar este manuscrito. Sin embargo, esto resulta confuso, ya que Mayhoff agrupó bajo esa misma sigla unas variantes señaladas por Detlefsen a partir de unos códices que no especifica.

${ }^{28}$ Citado únicamente en Reynolds 1983, sin designación previa latina. Antes conocido como Phillipps 8297 y Cheltenhamensis (Desanges 1980, p. 45).

${ }^{29}$ Desanges 1966, pp. 508-525.

${ }^{30}$ La designación $p$, usada en la edición de Les Belles Lettres, coincide con la asignada para el Pollinganus Latinus 11301 de Mayhoff. Para unificar de manera inequívoca pasamos a denominarlo como hiciera Sillig. 
Par Parisinus, códice que, según Harduino, estuvo en un colegio de la Sociedad de Jesús (recogido así por Sillig)

Snaken. Snakenburgianus, conjunto de lecturas que, según informa Si1lig, utilizó Enrique Snakenburg (Heinrich Snakenburg, 15741650) y anotó a mano en una ed. de Froben (Basilea, 1525)

Tomando en consideración la complejísima transmisión del texto pliniano, de la que no podemos dar cuenta en un trabajo de estas dimensiones ${ }^{31}$, señalaremos las relaciones entre los manuscritos aquí citados para dotar del debido rigor filológico al estudio. Para el mismo se han tenido en cuenta cerca de sesenta ejemplares, así como toda la información disponible en las ediciones y los estudios de crítica textual pliniana. Simplificando lo más posible, a una tradición más antigua ${ }^{32}$ remiten los manuscritos $E$ y $A$. Los demás se derivan de una tradición más reciente. Entre ellos, guardan estrecha relación $C h, R \mathrm{y}$ $D+G+V$ (seguramente producto de la división de un códice original). $F$ se relaciona con $G$, y son próximos a él $d$ y $T$. Con otro hiparquetipo se vincula $E$, y con él se relacionan $e, g, C o l b$ y, hasta cierto punto, $p$. Parecen descender de $E$ con algún testimonio intermedio los manuscritos $X$, Co y $o$ (mencionado en nota), mientras que $C, l, O x$ y $S$ tienen afinidad tanto con $E$ como con $o$. Por último, $a$ remite a otro hiparquetipo, dentro de este gran grupo de testimonios.

\section{Pervivencia sumergida}

Al estudiar las Obseruationes y confrontarlas con ediciones actuales, nos topamos con frecuentes casos en que su autoría está mal atribuida. Hemos contado más de cuarenta, entre aquellos que se adjudican indebidamente a Harduino (II 75, II 90, II 147, V 42, VII 23, X 37, XV 122), Gelenio (V 92, VIII 118, XII 65, XII 95, XIV 95), Sillig (V 21, V 103, VIII 92, XII 30, XII 95, XIV 68), a la ed. de Lion (III 9, X 120, XIII 77), Broterio (XII 135, XV

${ }^{31}$ Empleamos como base los datos manejados en Bran 2016 (tesis no publicada). Sobre ellos se han hecho las pertinentes aportaciones para el presente artículo.

32 Seguimos la distinción que apuntó Reynolds 1983, pp. 307-316, ya que en el caso de Plinio tiene, a nuestro juicio, más sentido que la tradicional división entre códices uetustiores y recentiores. 
77) Detlefsen (III 37, XIV 104), Ian (IX 185, XI 274) y, puntualmente, Müller (X 174) y Mayhoff (VI 31) ${ }^{33}$.

Queremos mencionar asimismo en este apartado la existencia de enmiendas en las que el Pinciano arregla acertadamente la ed. de Froben que le sirve de base. En muchos casos son correcciones, a su vez, a las Castigationes de Hermolao. Con frecuencia los editores siguientes no incluían de inmediato las propuestas de Núñez de Guzmán, por lo que las lecturas erradas aparecían de nuevo hasta corregirse después ${ }^{34}$.

\section{Grandes aciertos}

Bajo este epígrafe veremos una serie de casos en que la calidad de las $O b$ seruationes queda patente al haber perdurado en las ediciones actuales. Ello nos permite seguir acudiendo al Pinciano para tratar de solucionar los loci desperati que aún alberga el texto de Plinio. Sobre la base de diferentes argumentos (códices, ediciones, fuentes de Plinio, datos históricos, el usus auctoris o la sintaxis del texto), propone enmiendas que se han ido aceptando, por más que no siempre se hiciera de inmediato. En ocasiones se han producido hallazgos manuscritos recientes que han corroborado su lectura. El relieve auténtico de la labor del Pinciano se evidencia en estos diez casos concretos, que pasamos a comentar brevemente:

Plin., Nat. III 79 [Obseru. I 3, cap. 6, f. 9re.-ue.]: Sunt aliae uiginti ferme paruae mari uadoso, Galliae autem ora in Rhodani ostio Metina, mox quae Blascorum uocatur, et tres Stoechades a uicinis Massiliensibus dictae propter ordinem quo sitae sunt. nomina singulis Prote, Mese (...).

quo site sunt $F^{2}$ in ras., Pint. (e T!), Gel., Sil., edd.: quo sita erunt $d^{2}$ in ras., $T \mid$ quo sitae sunt erunt Snak., uet. a. Bar. | quos iterum $D F^{l} E$ a $\mid$ quas iterum Hard. (e codd.) | quos ita $d^{2} \mid$ quas item $R$ (?) Bar, L., Frob.

El Pinciano se encuentra en la ed. de Froben con una lectura que ve claramente corrompida, quas item nominant singulis uocabulis Proten. Nos

${ }^{33}$ Sus enmiendas a Pomponio Mela sufrieron un destino similar: la edición de Vosio es propensa a adjudicarse correcciones del humanista, lo que desemboca en el oscurecimiento efectivo de su autoría.

${ }^{34}$ Asís 1974 reivindicó este hecho en su trabajo, centrándose en Nat. II. 
centramos aquí en su reconstitución de quo site sunt, variante que únicamente se recoge en una segunda mano de $F$, tachada. Hasta el redescubrimiento de tal testimonio, solo contábamos con la defensa del Pinciano, que fueron aceptando los editores a partir de Gelenio. Si bien Hernán Núñez afirma basarse en $T$, en dicho material leemos de primera mano quo sita erunt, otra variante por lo demás bastante inusitada. Surge aquí la duda de qué habría sucedido realmente: cabe la posibilidad de que el Pinciano hubiera visto esa lectura en algún testimonio que no cite, acaso $S$, pues es el otro que emplea de forma sistemática. También podría ser una propuesta ope ingenii que hubiera querido dotar de un argumento de autoridad en el que apoyarse. Con todo, llama la atención el hecho de que coincida con un manuscrito. Nos inclinamos a pensar que, a partir del quo sita erunt de $T$, obtuvo la pista que le permitió descartar aquel quas item de Froben para reconstruir quo site sunt, sobre la base de posibles cortes erróneos. Otra ayuda para su propuesta sería la lectura común en las primeras ediciones impresas quo sitae sunt erunt: no deja constancia de si habría consultado alguna de ellas, mas esto no sería un procedimiento ajeno al humanista. Su propuesta, sin apenas apoyo documental, queda así como la canónica para la posteridad.

Plin., Nat. III 85 [Obseru. I 3, cap. 7, f. 9ue.]: Contra Vibonem paruae quae uocantur Ithacesiae ab Vlixis specula.

ab Vlixis Pint., Hard. e MCap., edd.: ab Vlyssis Ch| ab om. F RE $R^{2}\left(D R^{l}\right.$ a d T ulixes) $S$ uet., Frob.

El Pinciano es el primero en sugerir la adición de $a b$, para lo que se basa en las lecturas de Marciano Capela. Harduino, que también reconstruye el texto con dicha adición, afirma que debe al mismo Capela su enmienda y, en lo sucesivo, será la lectura aceptada. Podemos considerar la posibilidad de que Harduino desconociera la propuesta del Pinciano, habida cuenta de la proximidad de las fechas, pero es este quien la sugiere en primer lugar. Se trata, por lo demás, de una lectura ausente del grueso de manuscritos y de todas las demás ediciones del momento.

Plin., Nat. III 97 [Obseru. I 3, cap. XI, f. 10re.]: Oppidum Croto, amnis Neaethus, oppidum Thuri inter duos amnes Crathim et Sybarim, ubi fuit urbs eodem nomine.

Thuri Pint. (ap. May.!), Hard., Det., Ein.: -ii Pint., Rack., KW|-ium uet., Frob. | tyri $A \mid$ tauri $D F R E T S \mid$ taurium $a$ 
En este caso no hay consenso en las ediciones actuales, por más que la disensión sea mínima: bien Thuri (Detlefsen, Mayhoff, Einaudi), bien Thurii (Rackham, König-Winkler). Nuevamente, el Pinciano es el introductor de una enmienda, Thurii (Mayhoff da erróneamente Thuri) en la que difiere de su edición de base (Thurium) y de sus dos manuscritos en los que a menudo se apoya (tauri). El término aparece, por tanto, corrupto en todos los ejemplares, con una gran mayoría ofreciendo tauri. La base del Pinciano para su propuesta es el hecho de que Esteban de Bizancio llama a la misma ciudad Thurium, Thuria y Thurii. Gracias a sus profundos conocimientos propuso una variante distinta y de gran valor, en contra de todos los testimonios restantes.

Plin., Nat. III 112 [Obseru. I 3, cap. XIV, f. 10ue.]: Vmbrorum gens antiquissima Italiae existimatur, ut quos Ombrios a Graecis putent dictos, quod in inundatione terrarum imbribus superfuissent.

ombrios Pint., Gel., edd. (Det. ${ }^{2}$ ): umbrios uet. Frob., Det. $^{2} \mid$ umbrius $A \mid$ umbriuos $D$ FRE L d T | umbrinos $a$

Frente a umbrios de Froben y a umbriuos, predominante entre los manuscritos, opina el Pinciano que parece que deba leerse ombros u ombrios, a partir del término griego ombros 'lluvia', como encuentra en Esteban de Bizancio. Su lectura es única y gozaría de aceptación unánime en la posteridad, con la única excepción moderna de Detlefsen 1904. Su tratamiento como enmienda en las ediciones varía, desde el caso de Mayhoff, quien la reconoce en su aparato crítico, al de Zehnacker (Les Belles Lettres), quien no la consigna, por lo que es una variante correcta pero parcialmente oscurecida.

Plin., Nat. IV 22 [Obseru. I 4, cap. VI, f. 12ue.]: Peloponnesus in latitudinem a promunturio Maleae ad oppidum Aegium Corinthiaci sinus $\overline{C X C}$ patet, at in transuersum ab Elide Epidaurum $\overline{\mathrm{CXXV}}$.

Aegium $A$ E d g Colb., Par., Pint., Hard., edd.: regium $D T S \mid$ rhegium $F \mid$ rhegeum Snak., uet. | lechaeum Bar., Frob.

La enmienda Aegium, propuesta por el Pinciano, se recoge hoy en todas las ediciones del texto de Plinio como la idónea. El humanista se alejaba en gran manera de la lectura lechaeum de Froben, editor que, a su vez, la tomó de Hermolao. Tampoco le parecía adecuado el término que contenían sus dos códices, regium, que, si bien más cercano al auténtico, conservaba un error. $T$ y $S$ pudieron, sin embargo, ponerlo tras las huellas de la variante adecuada. 
En la actualidad contamos con evidencias manuscritas que refrendan la propuesta de Núñez de Guzmán. Lejos de quitarle valor, realzan el hecho de que fuera el primero en proponerla, incluso antes de contar con más apoyo. La explicación que dio el humanista partía de su conocimiento de la literatura clásica, en la que Egeo es una ciudad en el golfo de Corinto mencionada tanto por geógrafos como por Aristóteles o Séneca.

Plin., Nat. VI 181 [Obseru. I 6, cap. XXIX, f. 32re.]: Intrauere autem et eo arma Romana Diui Augusti temporibus duce P. Petronio, et ipso equestris ordinis praefecto Aegypti. Is oppida expugnauit (...).

aegypti. is Pint., Hard., edd.: aegyptiis D F R Frob. (e-), C. | aegyptus a d T (e-) p uet.

Nos encontramos ante un caso más de profundo conocimiento de las fuentes. Un aegyptiis que no parece encajarle en la sintaxis de la oración y un manuscrito toledano que tampoco da con la solución (aegyptus) conducen al Pinciano ante Estrabón como autoridad, quien ya hablara de un Petronio que fue prefecto de Egipto. La lectura no ha encontrado eco en ningún manuscrito de los empleados hoy y, sin embargo, se ha mantenido como canónica hasta la actualidad a partir de la ed. de Harduino.

Plin., Nat. IX 25 [Obseru. I 9, cap. VII, f. 55ue.]: pigeret referre, ni res Maecenatis et Fabiani et Flauii Alfii multorumque esset litteris mandata.

Fabiani $d$ (?) Pint., Hard., edd.: flauiani $F$ R a T uet., Frob.| flaui uet. ad indicem huius libri nr. 48 (ap. Sil.)

Recurriendo a Solino ${ }^{35}$ y a los índices de la Historia Natural, el Pinciano sugiere Fabiani donde antes se recogía flauiani, también en $T$. Ningún testimonio presenta solución similar, excepción hecha de la lectura de $d$, si bien solo la menciona Sillig y de manera dudosa. A partir de Harduino se acepta de forma general en las ediciones críticas, y por unanimidad en las actuales.

Plin., Nat. X 7 [Obseru. I 10, cap. III, f. 63ue.]: Phemonoe, Apollinis dicta filia, dentes esse ei prodidit, mutae alias carentique lingua, eandem aquilarum nigerrimam, prominentiore cauda; consensit et Boeus.

\footnotetext{
${ }^{35}$ Sol., XII 8: pigeret hoc adseuerare, ni Maecenatis et Fabiani multorumque praeterea esset litteris conprehensum.
} 
Boeus Pint. e Rom. ex., Hard., Det. ${ }^{2}$, Ern., KW: boethuius $F^{l} E^{1} \mid$ boethuus $E^{2} \mid$ boethus Bar., Frob., Det. ${ }^{1}$, Rack. | boethius $x$ e $l \mid$ boetius $R$ Ox V Sil. (e Dal.), uet. | boetis $d \mid$ boetus $T \mid$ Huius $a \mid$ poeta huius $F^{2} \mid$ poeta $f$ (ap. Dal.)

Los nombres propios, tanto personales como geográficos, han constituido los casos más claros de monstra Pliniana, un auténtico quebradero de cabeza para los editores. El nombre de Boeus adopta aquí prácticamente todas las variantes posibles, lo que se debe a la fusión con el huius que lo sigue, así como a una escritura poco clara. Boetius parece la opción predominante en las primeras ediciones, lo que a partir de las Castigationes de Hermolao (y, por consiguiente, también en Froben) quedó como boethus. En los casos más extremos se llegó a la pérdida del nombre propio y, al tener constancia de que se estaba refiriendo a algún autor, se añadió poeta, a partir del —oe- que se encuentra en el medio. Por otro lado, la lectura que Dalecampio atribuye al que tradicionalmente se ha denominado códice chiffletiano pierde huius y muestra tan solo poeta.

El Pinciano recurre para su enmienda a Ateneo, quien cita en su libro noveno a Boeo como autor de la Ornitogonía. Para terminar su explicación, el humanista reconoce que Boethus es un nombre frecuente en Grecia, pero Boeus no es inusitado: antes bien, lo menciona Pausanias a tenor de la fundación de Boeas, además de referir la presencia de una mujer divina llamada Boeona entre los fóquicos. Asimismo, añade, en los índices de los ejemplares romanos y de Parma aparece Boeus, y no Boethus.

Al tener únicamente dos manuscritos a su disposición, el Pinciano entabla su diálogo con las ediciones impresas que tiene ante sí, sus índices y los autores clásicos. Todo ello le valió para proponer una lectura que se acepta hoy por amplia mayoría: la única voz discordante es Rackham, que se adhiere a la opinión de Bárbaro y a la primera corrección de Detlefsen. Hernández Miguel 2003 , p. 356, aclara que el nombre de la sacerdotisa de Delfos era Boio, masculinizado después como Boîos y deformado en Plinio hasta Boethus.

Plin., Nat. X 155 [Obseru. I 10, cap. LV, f. 69re.]: super omnia est anatum ouis subditis atque exclusis admiratio prima non plane agnoscentis fetum, mox incerti singultus sollicite conuocantis, postremo lamenta circa piscinae stagna mergentibus se pullis natura duce.

incerti singultus Pint., Sil. (ex f), edd.: incertis incultus (-tuus $\left.D F^{I}\right) D F E^{2} d T e$ | incultis (-tos uet.) | incubitus $R$ x a l Ox V $E^{l}$ a, uet. | incerti incubitus Bar. e codd. | incerti concubitus Dal. (e uet.)| incertos incubitus Frob., Dal. et Brot. (ap. Sil.) 
Vuelve el Pinciano a aventurar una enmienda propia sin apoyo documental, presentada solo en las lecturas corruptas del llamado códice chiffletiano. Conllevaba entender un falso corte y la confusión de una $g$ por $c$, lo que la convertía en una propuesta un tanto arriesgada, mas hoy se acepta por delante de los demás manuscritos y sus muchas variantes, de las primeras ediciones impresas y de las enmiendas de otros estudiosos como Hermolao. Con el fin de poder descartar las lecturas de Froben (incertos incubitus) y de $T$ (incertis incultus), el Pinciano hace una propuesta en que recompone una gradación semántica ascendente en la cláusula: sorpresa inicial, sollozos dubitativos y lamentos.

Plin., Nat. XI 148 [Obseru. I 11, cap. XXXVII, f. 76ue.]: Media eorum cornua fenestrauit pupilla, cuius angustiae non sinunt uagari incertam aciem ac uelut canali dirigunt obiterque incidentia facile declinant, aliis nigri, aliis raui, aliis glauci coloris orbibus circumdatis.

raui, aliis $F^{2} R^{2}$ Pint., Gel.', edd.: araui $D F^{l} R^{I} \mid$ acra ui $E$ a $\mid$ atra uet. (atri Frob.) $\mid$ add. aliis rufa (rufi Frob., Gel.) uet. a. Hard. $\mid$ talis $F^{l} a \mid$ arauitalis (araui talis $T$ ?) $S$

Al toparse con un atri aliis rufi que no le da sentido, el Pinciano recurre a dos testimonios que, aunque errados, le sirven de pista para dar con la lectura correcta. $T$ presenta una extraña forma arauitalis (posiblemente en dos palabras, pues encontramos una pequeña separación) y, si obedecemos a la noticia del humanista, $S$ ofrecería similar solución. A partir de ahí, propone raui en lugar de atri y secluye aliis rufi. Esta compleja enmienda se vería posteriormente corroborada por segundas manos en $F$ y $R$. La seleccionan Gelenio y los editores actuales.

Mayhoff adjudica al Pinciano solamente la propuesta de raui y omite que, además, lo separó de aliis y eliminó el aliis rufi intermedio, con lo que ofrecía ya la lectura tal y como la encontramos en la actualidad. El valor de una enmienda tan compleja, máxime sin contar con manuscritos que la contuvieran, queda señalado al margen como locus eximius.

Además de los anteriores, hay otros pasajes aceptados hoy de los que queremos dar cuenta: diductus en lugar de deductus (Nat. III 119); la medida de $\overline{C C L}$ para el contorno del lago Mareotis (Nat. V 63), preferida a partir de Detlefsen, frente a los manuscritos $(C D)$ y las ediciones antiguas $(\overline{D C})$; la corrección Pariedros en lugar de Partedoros (Nat. VI 29), montes de los que se habla en Nat. VI 25; y la enmienda de corruptis por corrupti (Nat. XI 92), lectura que fue refrendada posteriormente por varios manuscritos $(F R a)$. 


\section{Enmiendas recuperables para su estudio}

Incluimos aquí una selección de enmiendas que se han silenciado, a nuestro juicio, indebidamente. Estamos ante pasajes sin una solución satisfactoria, que pueden adoptar nuevas posibilidades a raíz de un examen profundo de las Obseruationes.

Plin., Nat. V 9 [Obseru. I 5, cap. I, f. 18ue.]: Inde sinum qui uocetur Sagigi, oppidum in promunturio Mulelacha, flumina Sububam et Salat, portum Rutubis a Lixo CCXXIIII, inde promunturium Solis, portum Rhysaddir, Gaetulos Autoteles, flumen Quosenum, gentes Selatitos et Masatos, flumen Masathat, flumen Darat, in quo crocodilos gigni.

selatitos D Ch F E a C Co X h T Snak., edd.: selaticos $R \mid$ uelatitos $A$ Det. ${ }^{I} \mid$ scelatitos Frob. | salatitos Pint.

El Pinciano se separa de $T$ en la denominación de los llamados Salatitos y proporciona esta enmienda con la única base de una posible relación etimológica y de la colocación de elementos: en efecto, Plinio inserta el sintagma gentes Salatitos et Masatos entre una referencia al río Sala y otra al río Masathat. El río que el autor llama Sala se presenta en varios testimonios como Salat ( $A D F R E$, Ian y las ediciones actuales). Teniendo en cuenta que es perfectamente plausible la hipótesis de que Plinio se estuviera refiriendo a ese río cuando cita la población de los Salatitos (algo en lo que coinciden editores modernos), la propuesta del Pinciano constituye una forma más cercana a su nombre de origen $y$, por consiguiente, una enmienda que tener en cuenta.

Plin., Nat. VI 38 [Obseru. I 6, cap. XIII, f. 28re.]: Inrumpit autem artis faucibus et in longitudinem spatiosis atque, ubi coepit in latitudinem pandi, lunatis obliquatur cornibus, uelut ad Maeotium lacum ab ore descendens, sicilis, ut auctor est $M$. Varro, similitudine.

sicilis D F R E a g Colb., Par., S Pint., Hard., edd.: silicis T| scythici arcus uet., Frob. | sicilicis Pint. e Festo

Nuestro humanista encuentra en su edición de base scythici arcus 'arco escita', una lectura coincidente con las ediciones impresas de su momento. Esto parece un caso de corrupción por simplificación del texto, frente a un término, sicilis, -is 'hoz', que resulta muy poco frecuente en la literatura latina y que el editor podía no entender, pero que viene apoyado por un número nada desde- 
ñable de manuscritos ( $D F R E$ a $S$ ). Además, cuadra por su sentido con el símil que presenta Plinio: una curva del mar semejante a los cuernos de la luna. Como decíamos, el término solo aparece en Plinio (si aceptamos la lectura más extendida en los manuscritos) y en Ennio, toda vez que se trata de un pasaje que presenta disensiones y en que se alterna, precisamente, con sicilicibus en dos códices $^{36}$. A este respecto, Pablo el Diácono apunta en sus excerpta del De uerborum significatione que siciles son hastarum spicula lata 'anchas puntas de lanzas ${ }^{37}$ y propone tal lectura para Sexto Pompeyo.

Hernán Núñez tiene a su alcance tres variantes testimoniadas: por un lado, scythici arcus en Froben, que le salta a la vista como incorrecta y es a partir de la cual se propone la enmienda; por otro, lee silicis en $T$; y, finalmente, encuentra sicilis en $S$. Considera que la lectura de $T$ ha de ser más próxima a la original, pero no del todo satisfactoria, mientras que el apógrafo $S$ se le antoja commodius et minore a uero distantia. Con su bagaje en letras latinas y su conocimiento de obras de la Antigüedad clásica y tardía, el Pinciano acude al uso de sicilicis en Festo y se apoya en la coherencia del pasaje.

Si sicilis era un término poco frecuente, sicilicis resulta aún más inusitado. Encontramos únicamente el caso de Festo, 337M, ya que los otros son solo aparentes, resultado de la declinación de sicilicus, una unidad de medida para líquidos que encontramos en Plinio y en Volusio ${ }^{38}$. Incluso en Festo es un término dudoso: si bien responde a la lectura presente en la mayoría de los manuscritos $^{39}$, la ed. de Teubner prefiere siciles (Müller, p. $453^{40}$ ), haciéndolo coincidir con las anotaciones de Pablo el Diácono. Atendiendo a Labbé 1679 , s. u., se trataría de un vocablo que se identifica con el griego ó $\rho \beta \eta \lambda$ $\beta$, esto es, un cuchillo semicircular usado entre los trabajadores del cuero. Aquí se utiliza como término de comparación.

Si hacemos una síntesis de lo antedicho, la propuesta del Pinciano se presta a recibir el apoyo de dos argumentos que la hacen preferible al resto

${ }^{36}$ Enn., Ann. sed. inc. 479: incedit ueles uulgo sicilibus latis. Sin embargo, los códices $E$ y $L$ presentan sicilicibus (v. ed. de Skutsch).

37 Seguimos la interpretación de Ennio que hace Martos 2008, p. 240.

${ }^{38}$ Nat. XIII 93, XXXI 57; Volusio XXIX 2, XLI 4, XLIII 6, XLVIII 5, L 3 y otros. Lo volvemos a encontrar en autores posteriores, como Chacón 1608, p. 162. Como unidad de medida, es más frecuente el uso de sic(u)la, sigla o siclus.

39 Está en todos los códices salvo en uno, $M$, donde aparece silices.

${ }^{40}$ Citamos por la página de Müller según es convención (v. DGE). 
de opciones: por un lado, y dentro de lo desusado de los términos sicilis y sicilicis, la última es una lectio difficilior clara; por otro, es un vocablo que habría posibilitado una simplificación en dos vías, como silicis y como sicilis, ambas documentadas en manuscritos. Finalmente, no se consigna en ningún aparato crítico. Atendiendo a todas las razones dadas, constituye una propuesta sólida para el pasaje que tratamos.

Plin., Nat. VI 191 [Obseru. I 6, cap. XXX, f. 32re.]: Contra in Arabico latere Diaron oppidum esse eorum.

Diaron (dianon $\left.E^{S}\right) D F R E$ a T uet., Det., edd.: diaon $p \mid$ danion $S \mid$ daron Bar. e Ptol., Frob. | dalion Pint.

El Pinciano presenta un comentario en el que, como en otros lugares, critica la elección de Hermolao, pues es la lectura que recoge la ed. de Froben. Además, aporta una prior lectio ${ }^{41}$, Diaron, así como la lectura presente en $S$, Danion. Concluye que quizás ninguna de esas dos formas sea correcta, y propone en su lugar Dalion, al ser el nombre de un autor aducido por Plinio en otros lugares. El humanista prestaba particular atención no solo a elementos de léxico como tal, sino también a la coherencia del texto.

La propuesta Dalion aparece, en efecto, un poco después (Nat. VI 194) y permitiría una construcción paralela al Aristocreon ... tradit de la frase anterior $^{42}$, con elisión del verbo que no resulta ajena a la sintaxis rápida de Plinio. Por otro lado, de Diaron no hay más noticia en la literatura latina aparte de en este pasaje. Viene testimoniado en un elevado número de materiales, entre los que se incluye $T$, manuscrito que el Pinciano pasa aquí por alto, acaso porque no le aporta información relevante. Sin embargo, $S$, que cuenta con lecturas acertadas en otros lugares y que el Pinciano tiene en alta consideración, muestra otra variante, así como $p$. La presencia de hapax en la Historia Natural continúa planteando dilemas de compleja resolución.

Plin., Nat. VII 19 [Obseru. I 7, cap. II, f. 38re.]: hae sacrificio annuo, quod fit ad montem Soractem Apollini, super ambustam ligni struem ambulantes non

${ }^{41}$ Las designaciones del Pinciano no siempre son transparentes. En este caso, tras contrastar con varios incunables (como el parmense de Portilia 1481), podemos afirmar que se refiere a ediciones previas al trabajo de Hermolao.

${ }^{42}$ Aristocreon Libyae latere a Meroe oppidum Tollen dierum V itinere tradit. 
aduruntur et ob id perpetuo senatus consulto militiae omniumque aliorum munerum uacationem habent.

consulto F R a Frob., edd: consultu o T S Pint.

Nos encontramos ante una enmienda que no modifica el sentido del texto, pero que permite refinarlo para que resulte más ajustado al estilo de Plinio, además de constituir una anotación interesante del Pinciano en lo que respecta a la declinación de senatusconsultum como un vocablo unitario.

Como vemos, algunos manuscritos y la edición de Froben presentan senatus consulto, algo que vemos apoyado por los editores modernos sin excepción. La lectura en $-u$ traslada la declinación cuarta de senatus al último elemento del compuesto, que deja de entenderse como un adjetivo sustantivado de la segunda declinación, al ir prácticamente soldado con el sustantivo que lo precede. Dicha lectura estaba testimoniada en los manuscritos $S$ y $o$, y a ellos hay que añadir ahora $T$ (cotejado de primera mano). Por lo demás, el Pinciano aduce el hábito de escritura de Plinio, pues, según él, tiene costumbre de cambiar ciertos sustantivos a la cuarta declinación.

Consideramos que hemos de tener en cuenta esta posibilidad, ya que se encuentra testimoniada en autores antiguos y en manuscritos. Plinio solamente repite la expresión senatus consultum en I 8a, VIII 64, VIII 135, XXX 12 y XXXVI 85.

Plin., Nat. VII 123 [Obseru. I 7, cap. XXXVII, f. 42re.]: Hippocrates medicina, qui uenientem ab Illyriis pestilentiam praedixit discipulosque ad auxiliandum circa urbes dimisit, quod ob meritum honores illi quos Herculi decreuit Graecia.

discipulosque F R a edd.: et discipulos o, uet. a. Hard., Frob. | discipulis quos TS Pint.

El humanista parte de lo que él designa como uetera exemplaria para darnos una lectura (discipulis quos) que hoy en día no se consiga en las ediciones. Esta es una denominación vaga, pero el cotejo de manuscritos y ediciones nos lleva a confirmar que se trataría de lo que encuentra en los ejemplares $T$ y $S$.

En las primeras ediciones impresas, entre las que se incluye la de Froben, se lee et discipulos, que el Pinciano decide descartar y, con él, el resto de editores actuales, estos sobre la base de varios manuscritos a los que aquel no tendría acceso. Su intuición lingüística fue suficiente para percatarse de que se trataba de una lectura errada, y hace su nueva propuesta según lo que 
encuentra en sus apógrafos. Dicha propuesta resulta adecuada desde el punto de vista de la gramática y no es desatinada desde la crítica textual, si nos atenemos a las dimensiones y al parecido. Si acudimos, por fin, al sentido, sigue siendo plausible su aceptación: se entendería no ya que «Hipócrates predijo la peste procedente de los ilirios y envió a sus discípulos», sino más concretamente que «previno de la peste procedente de los ilirios a sus discípulos, a quienes ${ }^{43}$ envió a las ciudades vecinas para ayudar».

Plin., Nat. VIII 135 [Obseru. I 8, cap. XXXVII, f. 51ue.]: magnum fraus et ibi lucrum monopolio inuenit, de nulla re crebrioribus senatus consultis nulloque non principe adito querimoniis prouincialibus.

prouincialibus uet., Frob., edd.: promittialibus $\left(-t \mathrm{ttital}-R^{2}\right) R d \mid$ permittialibus $D F T$ | pernitialibus $E$ a $\mid$ pernicialibus $l T$ (ap. Pint.!) S Pint.

Nos encontramos de entrada con un error del Pinciano al leer (o quizás recoger) $T$. Nos indica que, a partir de uterque codex, ha de escribirse pernicialibus. Sin entrar en largas consideraciones acerca de la fiabilidad del humanista ${ }^{44}$, es posible que en este caso lea permittialibus (lo que realmente encontramos en $T$ ) como una simple corrupción por pernicialibus y que, por ello, no requiera de más explicación, al serle suficiente para rechazar prouincialibus. Suponemos, por lo demás, que en $S$ se recoge igualmente pernicialibus, con independencia de su grafía exacta: el uso de permities por pernicies, así como de permittialis, es frecuente en los manuscritos de Plauto, Tácito, Lucrecio o Plinio, y además es un uso que ya recoge Donato (Keil 1857, vol. IV, p. 392) (5) $^{4}$ La letra de $T$, en un estilo redondeado próximo al beneventano, no deja lugar a dudas en cuanto a la resolución de la abreviatura para per-. Una confusión entre prefijos (pro-/prae-/per-) sería habitual acaso entre editores, que tenían tendencia a suponer errores de ese tipo, mas no tanto entre escribas ${ }^{46}$. El Pinciano había de conocer tales usos a la perfección.

${ }^{43}$ También puede entenderse como «a los discípulos que envió...», suponiendo que no enviara a todos.

${ }^{44}$ Fue objeto de minuciosa comprobación en mi tesis doctoral, Bran 2016, pp. 249-266.

${ }_{45}$ Festa 1896, pp. 293-294.

${ }^{46}$ Un claro ejemplo de esta tendencia entre editores sería la sustitución de la lectura proriga (mayoritaria en los códices) por otras como perorigam o prurigam (Nat. VIII 156). Aunque poco frecuente (solo lo recogen Plinio y Varrón), su etimología parece remitir a pro-rego. 
La base definitiva para sugerir la sustitución por pernicialibus es el empleo del mismo término en Nat. XV 74. Esto es un auténtico alarde de agudeza y habilidad filológica, al valerse del usus auctoris de un caso puntual en una obra tan voluminosa. Añadimos otras muestras del uso de este adjetivo en VIII 13 (con proelia), XXII 94, XXIV 1 y XXXII 122. El término es mucho más raro que perniciosus, que es el verdaderamente usual en latín clásico, con casi cuatrocientas concordancias frente a seis de pernicialis, la mayoría en Plinio el Viejo. En el ThLL se consigna únicamente algún uso de Lucrecio junto a los de Plinio, con variantes gráficas pernici-/perniti-. Por otra parte, querimonia es una palabra que admite un uso no específico frente a otro técnico-jurídico, el de la queja presentada con carácter formal, a veces por los aliados (Cic., Div. Caec. 67, 7), otras por los ciudadanos (1. c., 9, 2). Este último es el sentido por el que se decantan los editores, y es perfectamente posible en Plinio. Sin embargo, la utilización normal como queja o lamento, con distintos adjetivos similares a pernicialis (malus, tristis, etc., cf. Hor., Carm. I 13, 19), es igualmente admisible.

La lectura que aceptan las ediciones actuales, prouincialibus, no cuenta con apoyo manuscrito, si bien es una reconstrucción que abunda desde las primeras ediciones impresas. En aquellas podría explicarse como una confusión paleográfica que se transmitiera de unas a otras. El contenido de este pasaje se refiere al uso de la piel del erizo, con sus púas, para cardar tejidos, y es en este sentido en el que aparece el fraus 'fraude' (bien por el monopolio en sí, bien por la calidad del tejido), sin que se mencionen provincias en ningún otro punto de esta sección. Los editores no pueden respaldar su lectura ni en los manuscritos ni en el usus auctoris, pues Plinio no utiliza querimonia en ningún otro lugar. La preferencia del Pinciano, por su parte, implica una lectio difficilior sin despojar de sentido al texto.

Plin., Nat. XII 29 [Obseru. II 12, cap. VII, f. 4ue.]: utrumque siluestre gentibus suis est et tamen pondere emitur ut aurum uel argentum. utrumque $R^{s}$ F E T Frob., edd: utcumque Pint.

En el comienzo de sus libros de botánica, Plinio inicia un recorrido por la India para detenerse en los pimenteros y se refiere, entre XII 27 y XII 30, a las varias clases de pimienta. En realidad, empieza distinguiendo tres tipos que son, más bien, tres fases distintas de un mismo producto. Así, el candidum piper 'pimienta blanca' es el que sale espontáneamente cuando madura 
al sol; el piper nigrum 'pimienta negra' es ese mismo, pero también secado al sol ${ }^{47}$; por fin, el bregma son las mismas semillas cuando se dejan a la intemperie, lo que produce cierta alteración en sus propiedades. Un poco más adelante menciona un piper longum 'pimienta larga', el más caro de todos. Plinio, que parece identificar de alguna manera las tres primeras clases, contrapone su sabor en una frase en que las diferencia con claridad ${ }^{48}$. Sin embargo, a la vez incluye informaciones sobre el zingiberi (o zimpiberi) ${ }^{49}$ y el Alexandrinum sinapi ${ }^{50}$. De ahí la dificultad inicial para los comentaristas y, entre ellos, para el Pinciano de interpretar utrumque, pues no está claro qué señala. Aunque Ernout 1949 lo traduce directamente como «le poivre et le gingembre», no deja de ser un pasaje de sentido dudoso, si no contradictorio, de cuya problemática Manzanero 2010, p. 31, n. 116, se ha hecho eco: «Expresión ambigua: $¿$ se refiere a la pimienta y el jengibre, o a la pimienta y la 'mostaza de Alejandría'?».

Sin apoyo documental, el Pinciano ofrece una solución: como bien nota, se habían indicado tres tipos de pimienta (larga, blanca y negra) y no dos, por lo que no cabe que diga utrumque 'uno y otro', y lee en cambio utcumque. Esta propuesta cuenta con varios argumentos a favor. A un nivel gráfico, es una hipótesis que se apoya en la fácil confusión de $c$ y $r$ en la cursiva. A un nivel de contenido, se pretende resolver la ambigüedad del texto de Plinio, pues este se podía referir a más de dos plantas. Por más que no podamos aceptarla indudablemente como la mejor lectura, sí consideramos que merece un lugar en las ediciones críticas.

\section{Nuevos planteamientos repugnantibus editionibus}

No queremos terminar sin dejar constancia de otras lecturas que subrayan aún más la labor que tenemos por delante cuando tratamos el texto de las Obseruationes, y que podrían ocupar nuevas empresas.

47 Plinio no identifica que se trata de la pimienta común, pero con su cascarilla (Manzanero 2010, p. 30, n. 109).

${ }^{48}$ Hoc [bregma] ex omni genere asperrimum est leuissimumque et pallidum, gratius nigrum, lenius utroque candidum (Nat. XII 27).

${ }^{49}$ De ambas maneras atestigua Plinio el jengibre.

50 'Mostaza de Alejandría' con la que se adultera la pimienta larga. 
Por una parte, nos topamos con propuestas que, si bien se han rechazado en las ediciones actuales, pues revisten una especial complejidad que nos impide su aceptación inmediata, son dignas de revisión. Se trata de flare en II 127, sitae en VI 25, la omisión de haec en VI 66, auorum para VII 50, diducta multitudine en XI 64 y la distinción de los tipos de pera Fauoniana y rubra para XV $54^{51}$.

Otras lecturas, a pesar de sus problemas, deberían recogerse en el aparato crítico, pues representan variantes distintas a las que se consignan en las ediciones y ofrecen nuevas posibilidades textuales: VI 79, VII 4, VIII 13, VIII 215, IX 166, XII 26, XIII 79, XIV 142.

\section{Conclusión}

La vigencia de las Obseruationes in loca obscura aut deprauata del Pinciano queda de manifiesto en nuestro estudio. En él hemos ubicado esa ingente tarea dentro del panorama específico de los comentarios españoles. Se ha procedido, asimismo, a apuntalar una identificación del manuscrito zaragozano.

En la sección central de este trabajo, y partiendo de un examen profundo de todas las ediciones y estudios a nuestro alcance, hemos comentado diez enmiendas que, con escaso apoyo manuscrito, han llegado como canónicas a las ediciones actuales, en una clara muestra del saber hacer del Pinciano, un reconocimiento a su labor. Se trata de Nat. III 79, III 85, III 97, III 112, IV 22, VI 181, IX 25, X 7, X 155, XI 148. Hemos apuntado, asimismo, otras cuatro relevantes. Al hilo de lo anterior, se proponen siete lecturas que hoy en día no se recogen en las ediciones críticas y que pueden usarse para solucionar pasajes complejos de Plinio. Dichas lecturas cobran nuevo sentido a la luz de nuestro conocimiento actual de los manuscritos y la crítica textual. Además, hemos puesto de relieve la diversa suerte de las enmiendas, que con cierta frecuencia quedan sumidas en el olvido de aparatos críticos que no las emplean de manera sistemática y que, en ocasiones, terminan por perpetuar una autoría errónea.

La figura de Hernán Núñez, que se ha ido recuperando en las últimas décadas, aún puede proporcionarnos útiles herramientas en virtud de la acri-

${ }^{51}$ Casi todas las variedades de esta fruta citadas por Macrobio encuentran su correspondiente aproximado en Plinio, lo que deja abierta la puerta a que este recogiera con rubrum el tipo de pera rubile de aquel. 
bia de su estudio, un saber hacer que se apoya tanto en los materiales que tenía a su alcance como en su sagacidad filológica.

\section{BIBLIOGRAFÍA}

Arrizabalaga, J. 2002: «Francisco López de Villalobos (c. 1473-c. 1549), médico cortesano», Dynamis 22, pp. 29-58.

Asís, M. D. de, 1974: El comendador griego Hernán Núñez de Guzmán 'El Pinciano' en la historia de los estudios clásicos, Salamanca.

Baranda, C. 2004: «La Celestina» y el mundo como conflicto, Salamanca.

Bárbaro, H. 1493: Castigationes Plinianae in Pomponium Melam, Roma.

Borst, A. 1994: Das Buch der Naturgeschichte, Heidelberg.

Bran, F. J. 2016: Plinio el Viejo en la España del siglo XVI: Francisco López de Villalobos y Hernán Núñez de Guzmán (El Pinciano), tesis doctoral, Universidad Complutense de Madrid.

Calamita, C. 1952: Figuras y semblanzas del Imperio: Francisco López de Villalobos, médico de reyes y príncipe de literatos, Madrid.

Chacón, P. 1608: Opuscula. In Columnae Rostratae inscriptionem, De Ponderibus, De Mensuris, De nummis, Roma.

Codoñer, C. 2011: «Las Castigationes del Pinciano a Plinio y a Pomponio Mela», Aevum 85, pp. 715-749.

Dalecampio, J. (ed.) 1587: C. Plinii Secundi Historiae mundi libri XXXVII, Lion.

Desanges, J. 1966: «Le manuscrit 'Ch' et la classe des recentiores perturbés de l'Histoire naturelle de Pline l'Ancien», Latomus 25, pp. 508-528.

Desanges, J. 1980: Pline l'Ancien. Histoire Naturelle, livre $V 1^{\text {re }}$ partie, 1-46 (L'Afrique du Nord), París.

Detlefsen, D. (ed.) 1866-1873: C. Plinii Secundi Naturalis historia, vols. I-VI, Berlín.

Detlefsen, D. 1869: «Die Naturalis Historia des Plinius. Die Indices der Naturalis Historia [Jahresbericht]», Philologus 28, pp. 284-337.

Einaudi, G. (ed.) 1982-1984: Storia Naturale, Turín.

Ernout, A. (ed. y trad.) 1949: Pline l'Ancien. Histoire Naturelle, livre XII, París.

Ernout, A. (intr. al vol. I) 1950: Pline l'Ancien. Histoire Naturelle, París.

Ernout, A. y Pépin, R. (eds. y trads.) 1947: Pline l'Ancien. Histoire Naturelle, livre $X I$, París.

Fabié, A. M. 1886: Vida y escritos de Francisco Lopez de Villalobos, Madrid.

Ferragut, M. C. 1993: El manuscrito de Juan Andrés Strany sobre la «Naturalis Historia» de Plinio (ms. de la biblioteca del Corpus Christi de Valencia) (tesis doctoral), Valencia.

Festa, N. 1896: «Della perpetua adolescenza d'Achille nell'Iliade», Rivista di Filologia e di Istruzione Classica 24, pp. 285-315. 
Friedenwald, H. 1939: «Francisco López de Villalobos: Spanish court physician and poet», Bulletin of the History of Medicine 7, pp. 1129-1139.

Gesner, C. (ed.) 1556: Claudi Aelianii... opera quae extant, Zúrich.

Granjel, L. S. 1979: Vida y obra de López de Villalobos, Salamanca.

Guzmán Arias, C. 1991: «Pervivencia de las aportaciones del Pinciano al "De Chorographia" de Pomponio Mela», en Ferreres, L. (ed.), Actes del IXè Simposi de la secció catalana de la SEEC. St. Feliu de Guíxols, 13-16 d'abril de 1988, Barcelona, vol. 1, pp. 411-419.

Harduino, J. (ed.) 1685: Caii Plinii Secundi Historiae naturalis libri XXXVII (5 vols.), París.

Hernández Miguel, L. A. (trad.) 2003: «Historia Natural. Libro X», en Moure, A. M. (coord.), Historia Natural. Libros VII-XI, Madrid.

Ian, L. von (ed. vol. 1) 1870: C. Plinii Secundi Naturalis historiae libri XXXVI, Leipzig.

Keil, H. 1855-1894: Grammatici Latini, 7 vols., Leipzig.

König, R. y Winkler, G. (intr., ed. y trad.) 1979-1997 (1l. I-XV): C. Plinius Secundus, der Ältere, Naturkunde, Múnich y Zúrich.

Labbé, C. 1679: Glossaria Latino-Graeca et Graeco-Latina, París.

Manzanero, F. (trad.) 2010: «Historia Natural. Libro XII», en Moure, A. M. (coord.), Historia Natural. Libros XII-XVII, Madrid.

Martos, J. (intr., trad. y notas) 2008: Ennio: Fragmentos, Madrid.

Mayhoff, K. (ed.) 1892-1909: C. Plinii Secundi Naturalis historiae libri XXXVII (1906 vol. I, 1909 vol. II, 1892 vol. III, 1897 vols. IV y V), Stuttgart.

Moure, A. M. 2008: «Plinio en España: panorama general», RELat 8, pp. 203-237.

Näf, B. (ed.) 2003: Roberti Crikeladensis Defloratio Naturalis Historie Plinii Secundi, Bergisch Gladbach.

Nauert, C. 1980: «Caius Plinius Secundus», en Kristeller, P. O., Kranz, F. E. y Brown, V. (eds.), Catalogus translationum et commentariorum: Medieval and Renaissance Latin translations and commentaries, Washington, vol. 4, pp. 297-422.

Núñez de Guzmán, H. 1544-1545: Obseruationes in loca obscura aut deprauata historiae naturalis C. Plinii, 3 vols., Salamanca.

Rackham, H. (ed. y trad.) 1938: Naturalis Historia, Londres-Cambridge.

Ramos, S. 2001: «¿Escribió Plinio el Viejo una enciclopedia?: A propósito de una lectura de Lucio Flaminio Sículo», en Alvar, A. y García Jurado, F. (eds.), Actas del XI Congreso Español de la Sociedad de Estudios Clásicos (21-25 de septiembre de 1999), Madrid, vol. 2, pp. 605-613.

Ramos, S. 2006: «Tradición pliniana en la Andalucía del siglo XVI: a propósito de la labor filológica del Doctor Francisco Hernández», en Rodríguez-Pantoja, M. (ed.), Las raíces clásicas de Andalucía. Actas del IV congreso Andaluz de Estudios Clásicos (Córdoba, 2002), Córdoba, pp. 883-891. 
Reeve, M. D. 2006: «Manuscripts of Pliny's Natural History in Spain», Exemplaria Classica 10, pp. 151-186.

Reeve, M. D. 2007: «The editing of Pliny's Natural History», Revue d'histoire des textes 2, pp. 107-180.

Reynolds, L. D. 1983: Texts and Transmission, Oxford.

Rodríguez-San Pedro, L. E. 2012: «Diego de Covarrubias en la Universidad Salmantina del Renacimiento», en Pérez, I. y Becedas, M. (coords.), Diego de Covarrubias y Leyva: el humanista y sus libros, Salamanca, pp. 69-91.

Serbat, G. 1995: «Introducción general», en Moure, A. M. (coord.), Plinio el Viejo. Historia Natural, libros I-II, Madrid.

Signes, J., Codoñer, C. y Domingo, A. 2001: Biblioteca y epistolario de Hernán Núñez de Guzmán (el Pinciano): una aproximación al humanismo español del siglo XVI, Madrid.

Sillig, J. (ed. y com.) 1831-1836 y 1851-1856: C. Plinii Secundi Naturalis historiae libri XXXVII, Leipzig.

Somolinos, G. y Nogués, M. del C. (intr. y ed. facsímil) 1998: F. Hernández. Historia Natural de Cayo Plinio Segundo (ca. 1560-1600), México.

Torre de Rezzonico, A. J., Conde de la, 1763: Disquisitiones Plinianae in quibus de utriusque Plinii patria, rebus gestis, scriptis, codicibus, editionibus atque interpretibus agitur, Parma.

Tremallo, B. S. 1991: Irony and self-knowledge in Francisco Lopez de Villalobos, Harvard dissertations in Romance languages, Nueva York-Londres.

Walter, H. 1996: «La tradizione manoscritta della Storia naturale di Plinio il Vecchio in età umanistica: il caso del codex Chiffletianus», Studi Umanistici Piceni 16, pp. 175-197.

Zehnacker, H. (ed. y trad.) 2004: Pline l'Ancien. Histoire Naturelle, livre III, París.

Fecha de recepción de la primera versión del artículo: 16/02/2020

Fecha de aceptación: 16/04/2020

Fecha de recepción de la versión definitiva: 29/06/2020 